\title{
INHALT.
}

Einleitung . . . . . . . . . . . Seite

Erstes Kapitel.

Berlin, Leipzig, Wien.

I. Berlin, gegen Breslan, Magdeburg, Hamburg, Posen, Potsdam:

II. Leipzig, Lokalpartie; ferner gegen Altenburg, Lübeck, Magde-

III. Wion, gegen Insterburg, Berlin: Partie 21-24.

Zweites Kapitel.

\section{Norddeutsche Schachkreise.}

I. Magdeburg, Potsdam, Partie 25-26.

II. Hamburg, Lübeck, Śchleswig otc., mit Glückstadt, Breslau etc: Partie 27-31. . . . . . . .

III. Hannover, Coile, Emden, Stettin etc.; mit Otterndorf, Dannenberg, Lüchow, Vegesack, Stargardt: Partie 32-40.

IV. Ost- und Westpreussen. Marienwerder-Kanitzken, KurnikSchrodo; Königsberg, Tilsit, Danzig, Thorn, Marienwerder, Insterburg, Gumbinnen: Partie 41-48.

$$
\text { Drittes Kapitel. }
$$

\section{Mitteldeutsche Schachkreise.}

Sachsen, Thüringen, Hessen etc. Zwischen Bautzen, Freiberg, Rochlitz, Gotha, Coburg, Langensalza, Nordhausen, Bernburg, Stollberg, Chemnitz, Penig, Lommatzsch, Bautzen, Görlitz, Nädlitz, Hanau, Schlüchtern : Partie 49-61. . . 40-48 Viertes Kapitel.

Siiddentsche Schachkreise.

Bayern, Württemberg, Baden. Zwischen München, Augsburg, Stuttgart, Carlsruhe, Frankfurt a M., Mannheim, Barmen: Partie 62-67.

F'ünftes Kapitel.

\section{Westdeutsche Schachkreise.}

I. Crefeld, gegen Elberfeld, Breslau, Amsterdam, Wesel, Düsseldorf: Partie 68-76. . . . . . 52-57

II. Elberfeld, gegen Aachen, Amsterdam: Partie 77-79. $:$. $57-58$

III. Düsseldorf, Barmen etc., mit einander, ferner Barmen, Duisburg, Lennep, Iserlohn, Ruhrort, Mülheim: P. 80-88. 60-G4 


\section{VI \\ Sechstes Kapitel. \\ Holland und Belgien.}

Amsterdam, gegen Rotterdam, Antwerpen, Haag, Zeijst, London;

Seite ferner zwischen Nymegen, Haag, Gouda, Rotterdam, Tiel, Zaltbommel, Arnheim, Leiden, Sneek, Leeuwerden: Partie 89-104. . . . . . . . . . . . . . . . . 65-77

Siebentes Kapitel.

\section{Frankreich.}

Paris gegen London, Pest; ferner zwischen Valenciennes, Douai, Orleans, Marseille, Algier: Partie 105-113.

\section{Achtes Kapitel.}

\section{England.}

I. London, Edinburg etc. Partie 114-120.

II. Cambridge, Oxford, Reading etc., mit Nottingham, Oxford, Stourbridge, Penzance, Preston, Hull, Lancaster: Partie 121-127.

III. Loeds, Liverpool, Birmingham, Nowcastle etc. ferner Armagh, Maryport, Inverness, Wakefield, Huddersfield, Nottingham, Glasgow, Berwick, Bristol, Worcester, Windermere. Partie 128-136.

Neuntes Kapitel.

\section{Schweden and Russland.}

Gothenburg, Stockholm, Upsala, Malmoe, Lund. - Arensburg, Dorpat: Partie 137-142. . . . . . . . . . . 108-111

\section{Zehntes Kapitel.}

Indien, Amerika, Australien.

Madras, Hyderabad, Shottisbrooke, City, „Hills" u. „Plains"; Norfolk, New-York, Philadelphia, Boston, Chicago, Quincy, Providence; - Georgetown, Hobarttown, Launceston; - Grahamstown, Uitenage: Partie 143-158. . . 112-122

\section{Schluss.}

\section{Nachträge und Uebersichten.}

Partie 159. München gegen Mannheim . . . . . . . . . 124

160. Mannheim gegen München . . . . . . . . . . 124

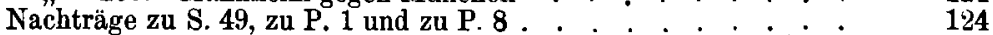

Correspondenzpartien, Berathungspartien, Drahtpartien . . . . 125

Partie 161. H. Staunton gegen Bristol . . . . . . . . . . . 126

" 162. L. Paulsen gegen Quincy . . . . . . . . . . . . . . 126

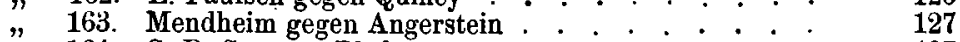

"164. C. R. S. gegen Bledow . . . . . . : . : . 127

Uebersicht von Correspondenzpartien zwischen einzelnen Schachfreunden . . . 127-128

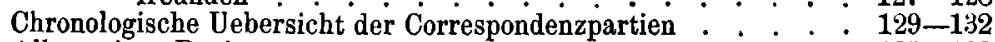

Allgemeines Register . . . . . . . . . . . . . . . . 133-138 